M. WETSS: Neue Harnproben. Demonstration. I. Eines in der Kälte und mit einem Tropfen Urin ausführbaren Zuckernachweises mit Hilfe eines haltbaren Pulvers "Glykostat". Ein Tropfen Harn wird auf einem Filtrierpapier mit einer kleinen Menge Glykostat gemischt. Bei positivem Ausfall der Reaktion Schwarzfärbung. 2. Einer modifizierten Benzinprobe. 3. Einer Modifikation des Histidinnachweises im Harn von KAPELLER-ADLER. Diese Reaktion ermöglicht eine schnelle und sehr frühzeitige Schwangerschaftsdiagnose. Auch bei perniziöser Anämie und bei manchen Fällen von Leberkrankheiten wird vermehrtes Histidin im Harn gefunden.

F. FLEISCHNER: Über das Wesen der basalen horizontalen Schattenstreifen im Lungenfeld. Bei abdominalen Erkrankungen, insbesondere bei Pankreatitis, werden ofters horizontale Schattenstreifen im basalen Lungenfeld gefunden, welche früher als Zeichen einer Durchwanderungspleuritis gedeutet wurden. Meist handelt es sich hierbei um alte und durch das Grundleiden geschwächte $\mathrm{Pa}$ tienten, bei welchen die Vitalkapazität der Lunge stark herabgesetzt ist. Die Vermutung des Vortr, daß $\mathrm{B}$ es sich hier um eine Resorptionsatelektase handelt, konnte autoptisch bestätigt werden. Es handelt sich um einen fächerförmigen Kollaps kleinerer Lungenpartien, dessen horizontale Ausbreitung durch die Richtung der elastischen Fasern bestimmt wird. Nach $\mathrm{CO}_{2}$-Atmung erfolgt Entfaltung der kollabierten Partien und daher radiologisch feststellbare Aufhellung.

J. FREUNDLICH: Ûber die Bedeutung der negativen Nachschwankung bei Rechts- und Linksform des Kammer-Elektrokardiogramms. An Hand eines größeren statistischen Materials wird festgestellt, daB auch beim Uberwiegen einer Kammer einer Negativität der Nachschwankung in einer oder zwei Ableitungen eine üble prognostische Bedeutung zukommt. I25 Patienten mit Linksüberwiegen und negativer T-Zacke in Abl. I wiesen innerhalb von 2 Jahren eine Mortalität von $40,5 \%$ und eine rezidivierende Herzinsuffizienz in $94 \%$ der Fälle anf. Von jenen Patienten, welche in 2 Ableitungen eine negative Nachschwankung hatten, zeigten roo\% chronische Herzinsuffizienz, die Mortalität betrug $56 \%$. Demgegenüber zeigten die Patienten mit Überwiegen einer Kammer bei positiver T-Zacke in allen 3 Ableitungen ein verhältnismäßig günstiges Verhalten.

D. SCHERF und H. SIEDECK: Ưber die Chininwirkung auf die Extrasystolen. Experimentelle Untersuchungen am Hundeherzen, bei welchem sich durch Applikation von Aconitin gehäufte Extrasystolen hervorrufen lassen, welche regelmäBig $1 / 2-$ I Stunde anhalten und meist in Form einer Bigeminie oder Trigeminie auftreten. Bei der Beeinflussung der Aconitin-Extrasystolen durch Chinin können 3 Wirkungstypen beobachtet werden, und zwar 1 . ein gänzliches Aufhören der Extrasystolen. 2. Ein Fehlen jeder Beeinflussung. Diese beiden Formen entsprechen der klinischen $\mathrm{Be}$ obachtung, welche ebenfalls zeigt, daß manche Extrasystolien auf Chinin ausgezeichnet ansprechen, während andere Formen unbeeinflußt bleiben. An den mit Aconitin vorbehandelten Herzen wird noch ein dritter Wirkungstypus beobachtet, bei welchem nach der Chininapplikation eine Häufung der Extrasystolen auftritt. Eine solche „paradoxe Wirkung" auf die Extrasystolen am Aconitinberzen kommt auch beim Kalium vor, welches Extrasystolen im allgemeinen behebt, am Aconitinherzen aber die Extrasystolie noch verstärkt.

Ausprache: F. Dressler. - A. Neumann. - K. F. Wenckebach. - D. SCHERF (Schlußwort). E. LAUda.

\title{
P. E. POULSSON
}

Am I9. Màrz I935 verschied in Oslo unerwartet aus voller Tätigkeit - wohl in einem Anfall von Angina pectoris - der norwegische Pharmakologe Poul EDVARd Poulsson gegen Ende seines 77. Lebensjahres. Nicht nur wegen der Stellung dieses Mannes in seinem Fach und damit in der internationalen Wissenschaft gebührt es sich, seines Scheidens zu gedenken, sondern noch im besonderen wegen des Einflusses, den er auf Generationen deutscher Ärzte ausgeübt hat durch sein sehr beliebtes „Lehrbuch der Pharmakologie für Ärzte und Studierende". Zwischen Igog und I933 erschienen 9 Auflagen in deutscher Sprache. Dies Buch zeichnet sich aus durch treffsichere Auswahl des umbedingt Notwendigen und möglichst Gesicherten aus dem reichen und fließenden Tatsachen- und Gedankengebiet der pharmakologischen Wissenschaft und durch eine knappe, dabei klare und leicht lesbare Darstellung. Was manchen Kenner unbefriedigt lieb, die Ignorierung interessanter Probleme und die vereinfachende Behandlung schwieriger Fragen, gerade das zog den Lernenden an, der ja zunächst das Bedürfnis nach grundsätzlicher Orientierung uber das ihm fremde und in seiner Fülle verwirrende Material empfindet.

Diese Haltung Poulssons in seinem Lehrbuch war offensichtlich bewußt und gewollt, wie der Fachmann an der Art erkennen konnte, wie er das Buch für die neuen Auflagen revidierte. Selbst auf Gebieten, wo er die Probleme durch eigene Arbeit besser kannte als jeder andere, blieb er im Lehrbuch zurtickbaltend und so sparsam an Worten wie möglich. Gewiß war dieser Grundsatz vom Standpunkt des erfahrenen Lehrers weise, wenn man auch mit Racksicht auf die Nachbardisziplinen der Klinik und der Pathologie den Rahmen des Buches als etwas enggespannt empfinden muBte.

Poulsson selbst war alles andere als ein enger Geist. Sein Wissen basierte auf einer ausgezeichneten naturwissenschaftlichen Schulung, vor allem in Botanik und Chemie, auf einem lebenslang geübten eifrigen Studium der wissenschaftlichen Literatur, endlich aber auch auf der täglich erneuerten Erfahrung in eigener ärztlicher Praxis. Utberdies blieb er natürlich der wissenschaftlichen Forschung unabänderlich zugetan, über alle amtlichen und ärztlichen Verpflichtungen hinweg bis zum letzten Tage seines Lebens.

Poulsson war geboren am I8. April 1858 in Larvik als Sohn eines Arztes. Entsprechend den Gewohnheiten und Vorschriften seines Heimatlandes verbrachte er viele Jahre mit seinem medizinischen Studium in Oslo, das er als Siebzehnjähriger begann. Nach weiterer klinischer Ausbildung kam er 1887 nach Deutschland; um zunächst bei FresenIUS in Wiesbaden seine chemischen Kenntnisse weiter zu entwickeln und im folgenden Jahr für $2^{2} / 2$ Jahre an die damals berühmteste Ausbildungsstätte für junge Pharmakologen zu gehen, nämlich an das Institut von Oswald SCFMTEDEBERG in Straßburg, dessen Assistent er im letzten Jahre seiner dortigen Wirksamkeit wurde. r8gr übernahm er dann die
Lehrstelle für Pharmakologie an seiner heimischen Universität Oslo, die er von 1895 an als außerordentlicher, von I9r3 an als ordentlicher Professor bis zu seinem 7o. Lebensjahr 1928 führte. $\mathrm{Zu}$ seinen Aufgaben gehörte die Einrichtung eines experimentellen Laboratoriums, dem er den Stempel seiner organisatorischen Kraft aufdrückte. Auch nach Entbindung von seinen akademischen Verpflichtungen ruhte sein Betätigungsdrang nicht. Er regte die Gründung eines staatlichen Vitamininstituts an, das in Sköyen bei Oslo errichtet wurde und das er bis zu seinem Tode leitete. Auch hier verband er das praktische Interesse, das in der wirtschaftlichen Bedeutung des Lebertrans für Norwegen liegt, mit dem theoretischen Interesse des Forschers an den Problemen der Vitaminwirkung und Vitaminbildung. Seine Arbeiten auf diesen Gebieten bewiesen die unveränderliche Frische und Beweglichkeit seines Geistes bis in ein Alter, das eine heute besonders beliebte Auffassung schlechthin als unproduktiv anzusehen pflegt.

In seinen fraheren Arbeiten beschäftigte sich Poulssos mit mannigfachen Einzelfragen seiner Wissenschaft; seine Feststellungen waren stets gründlich und gewissenhaft, bewährten sich daher für die Dauer und wurden Bausteine der anerkannten Lehre, so auf dem Gebiete des Strychnins, Cocains, der Farnwurzel, des Bariums, Quecksilbers und mancher anderer Kapitel. Seine zahlreichen zusammenfassenden Darstellungen bewiesen stets die gleichen Eigenschaften, wie sie seinem Lehrbuch das Gepräge gaben, vorzügliche Sachkenntnis und ansprechende Form, auch treffsichere Anpassung an den $Z$ weck der Abhandlung, mochte es sich nun um streng wissenschaftlich-theoretische Handbuchartikel, um Aufklärung der ärztlichen oder einer breiteren Öffentlichkeit, um Denkschriften für gesetzgeberische Instanzen oder sonstige Anlässe handeln. Besondere Erwähnung verdienen Poulssons Bemühungen um Abwehr der Homöopathie und sonstiger Abarten der Medizin, wie auch um Herbeiführung vernünftiger Verhältnisse auf dem Arzneimittelmarkt seines Landes; seine Vorschläge zur Regelung der Spezialitätenfrage u. dgl. sind vorbildlich.

Persönlich war Poursson von imponierender Statur und vornehmer Haltung, dabei liebenswürdig und von Esprit und Humor erfüllt. Drum war er auch überall in den deutschen Instituten seiner Fachgenossen und auf ihren Tagungen ein besonders gern gesehener und geschätzter Gast. Seiner Bedeutung und seiner persönlichen Beliebtheit Ausdruck war es, daß PouLsson mehrere Jahre im Vorstand der Deutschen Pharmakologischen Gesellschaft mitwirkte. So wird die Spur von seinen Erdentagen noch ferner eingezeichnet bleiben nicht nur im Verstande seiner deutschen Schüler, denen er das Erbe seines deutschen Lehrers ScrmiedeBERG fortgestaitend weitergab, sondern auch in den Herzen derer, die ihn kannten. WOLFGANG HeUBNer. 